\title{
High-Frequency Oscillatory Ventilation for Rescue From Refractory Hypoxemia in a Patient With Transfusion-Related Acute Lung Injury
}

\author{
Chin-Hua Huang, Han-Chung Hu MD, Meng-Jer Hsieh MD, Ching-Tzu Huang, Hsiu-Ying Cho, \\ Hsiu-Feng Hsiao MBA, Cheng-Ta Yang MD, Ying-Huang Tsai MD, \\ Chung-Chi Huang MD, and Kuo-Chin Kao MD
}

\begin{abstract}
Transfusion-related acute lung injury is a serious complication of blood transfusions. Herein is a report on a 32-year-old woman who developed diffuse pulmonary infiltrates and acute respiratory compromise after blood transfusion. Non-cardiogenic pulmonary edema was diagnosed based on data calculated by the hemodynamic monitoring system, but severe hypoxemia persisted despite conventional pressure-control ventilation with $100 \%$ oxygen, low tidal volume, and high PEEP. The refractory hypoxemia was improved by high-frequency oscillatory ventilation. This experience suggests that high-frequency oscillatory ventilation may be beneficial for patients with transfusionrelated acute lung injury and severe refractory hypoxemia. Key words: high-frequency oscillatory ventilation; refractory hypoxemia; transfusion-related lung injury. [Respir Care 2012;57(5):798-801.

(C) 2012 Daedalus Enterprises]
\end{abstract}

\section{Introduction}

Transfusion-related acute lung injury (TRALI) is a rare but life-threatening complication of blood transfusions. It usually occurs with the development of acute respiratory distress, non-cardiogenic pulmonary edema, and hypoxemia during or within 6 hours of transfusion. ${ }^{1}$ Treatment is mainly supportive, including oxygen support, noninvasive ventilation, or intubation with conventional mechanical ventilation. $^{2}$

The authors are affiliated with the Department of Respiratory Therapy, Chang Gung Memorial Hospital, Chang Gung University College of Medicine, Taoyuan, Taiwan. Han-Chung Hu MD, Meng-Jer Hsieh MD, Cheng-Ta Yang MD, Ying-Huang Tsai MD, Chung-Chi Huang MD, and Kuo-Chin Kao MD are also affiliated with the Departments of Thoracic Medicine and the Department of Respiratory Care, Chang Gung University College of Medicine, Taoyuan, Taiwan.

The authors have disclosed no conflicts of interest.

Dr Kao presented a version of this paper at the 56th International Respiratory Congress of the American Association for Respiratory Care, held December 6-9, 2010, in Las Vegas, Nevada.

Correspondence: Kuo-Chin Kao MD, Department of Respiratory Care, Chang Gung University College of Medicine, 5 Fu-Shing Street, KweiShan, Taoyuan, Taiwan, E-mail: kck0502@adm.cgmh.org.tw.

DOI: $10.4187 /$ respcare.01355
High-frequency oscillatory ventilation (HFOV) is an alternative mechanical ventilation used as rescue therapy for adult patients with acute lung injury (ALI) and acute respiratory distress syndrome (ARDS). Some observational studies have shown improved oxygenation in patients with refractory hypoxemia. ${ }^{3,4}$ A recent Cochrane review concluded that HFOV may be a rescue therapy for improving survival in ARDS patients. ${ }^{5}$

Herein is a case of TRALI with persistent hypoxemia under pressure-control ventilation mode with $100 \%$ oxygen, low tidal volume, and high PEEP. The refractory hypoxemia was effectively corrected by shifting to HFOV.

\section{Case Report}

A 32-year-old woman with insulin-dependent diabetes mellitus, hypertension, and chronic renal insufficiency was admitted to the gastroenterology ward due to hematemesis after intermittent nausea and vomiting for 5 days. Diagnostic esophagogastroscopy revealed gastroesophageal reflux and possible Mallory-Weiss tear. Before admission, no aspiration episode was described by the patient. After treatment with intravenous omeprazole $40 \mathrm{mg} / \mathrm{d}$, the hematemesis stopped 2 days later. She was transferred to the nephrology ward for progressively elevated blood urea nitrogen and serum creatinine. Before admission, the baseline serum blood urea nitrogen, creatinine, and daily urine 


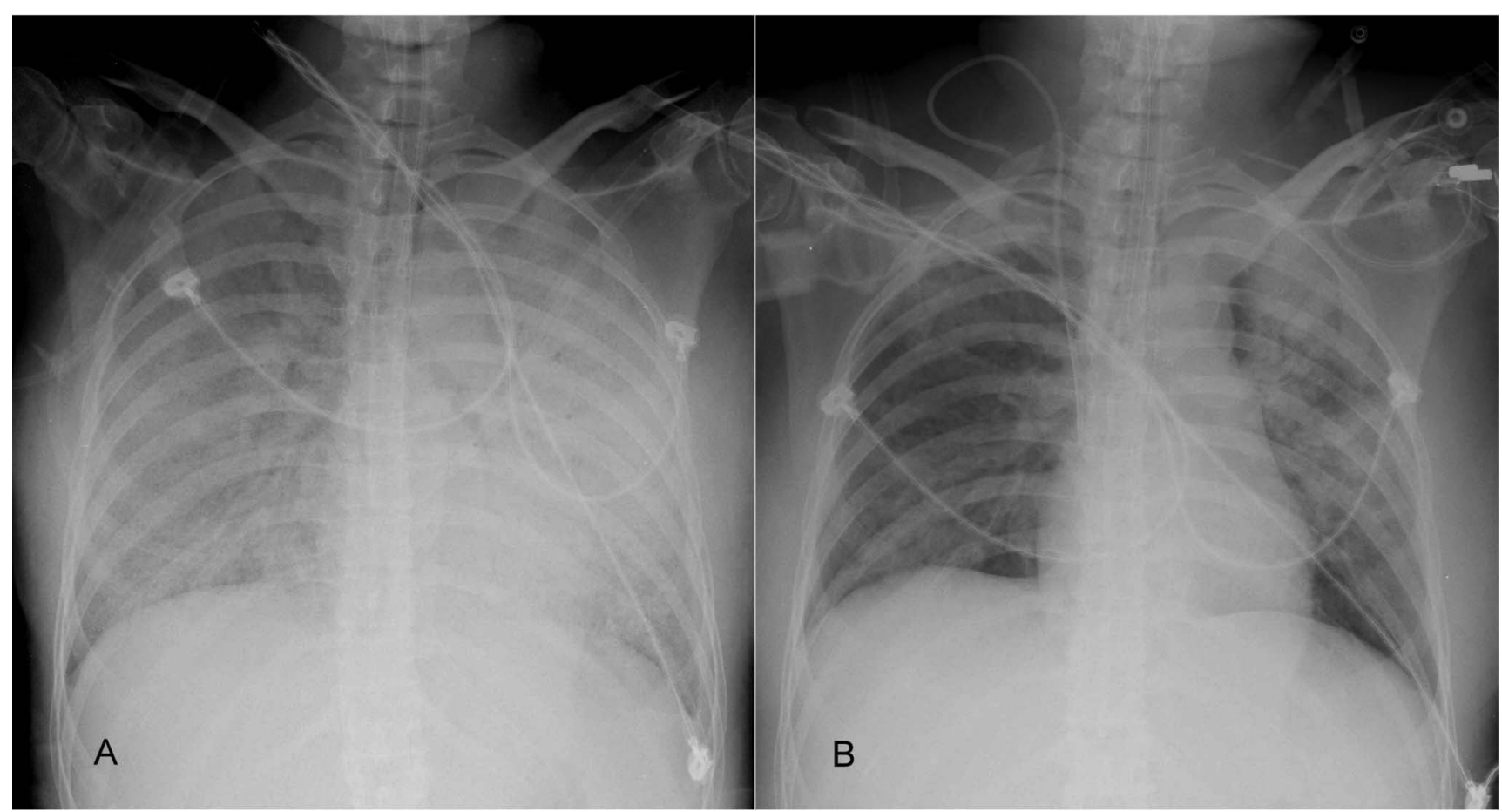

Fig. 1. A: Diffuse bilateral pulmonary infiltration indicating acute pulmonary edema after intubation. B: Improvement of the diffuse bilateral pulmonary infiltrates after 2 days of high frequency oscillatory ventilation (HFOV).

amount were $52 \mathrm{mg} / \mathrm{dL}, 4.1 \mathrm{mg} / \mathrm{dL}$, and 1,000-1100 mL. Compared with baseline renal function, there was no sudden decline in renal function on admission. Her serum blood urea nitrogen and creatinine were $55 \mathrm{mg} / \mathrm{dL}$ and $4.2 \mathrm{mg} / \mathrm{dL}$, respectively, on admission, but increased to $92 \mathrm{mg} / \mathrm{dL}$ and $5.0 \mathrm{mg} / \mathrm{dL}$, respectively, after 3 weeks. Under the diuretics treatment with oral furosemide $80 \mathrm{mg} / \mathrm{d}$, her daily urine amount was between $950 \mathrm{~mL} / \mathrm{d}$ and $1,150 \mathrm{~mL} / \mathrm{d}$ during admission. Suggested arteriovenous shunt creation for renal replacement therapy was declined.

Because of anemia (hemoglobin $7.5 \mathrm{~g} / \mathrm{dL}$ ), 2 units of packed red blood cells (packed RBC) were transfused, without adverse reaction. Chest radiograph taken the following day was unremarkable. Her hemoglobin was $7.8 \mathrm{~g} / \mathrm{dL} 6$ days after the first blood transfusion, so another 2 units of packed RBC were transfused to keep the hemoglobin above $8.0 \mathrm{~g} / \mathrm{dL}$. During these 6 days no other acute event or ongoing hemorrhage occurred. Thirty minutes after completing the transfusion, she experienced shortness of breath and her respiratory rate increased to 24 breaths/min. At the time of symptom onset, heart rate was 92 beats/min and blood pressure was 148/86 mm Hg. Bedside echocardiography revealed adequate left ventricle systolic function, with ejection fraction $66 \%$ by $\mathrm{M}$ mode and normal early wave/atrial wave $(\mathrm{E} / \mathrm{A})$ ratio $(\mathrm{E}>\mathrm{A})$ of mitral valve flow by Doppler. The serum troponin-I and creatine kinase-MB fraction level were within normal range $(0.11 \mathrm{ng} / \mathrm{mL}$ and $1.7 \mathrm{ng} / \mathrm{mL})$.
On physical examination there were fine inspiratory crackles in both lungs. Initial examination of arterial blood gases revealed $\mathrm{pH} 7.38, \mathrm{P}_{\mathrm{aO}_{2}} 66 \mathrm{~mm} \mathrm{Hg}$, and $\mathrm{P}_{\mathrm{aCO}}$ $32 \mathrm{~mm} \mathrm{Hg}$ on $\mathrm{F}_{\mathrm{IO}_{2}}$ of 0.5. In the 6 days between transfusions there was no mental change or aspiration episode. Because the respiratory distress did not respond to intravenous furosemide, she was intubated for mechanical ventilation. Post-transfusion hemogram showed hemoglobin $12 \mathrm{~g} / \mathrm{dL}$, with white cell count of $7,300 / \mathrm{mL}$. The discordant high concentration level of hemoglobin was caused by volume contraction due to diuresis. Chest radiograph after intubation revealed diffuse bilateral pulmonary infiltrates, indicating acute pulmonary edema (Fig. 1A).

Even under pressure control ventilation with $100 \%$ of oxygen, the patient had a low $\mathrm{S}_{\mathrm{pO}_{2}}$ of approximately $80 \%$. Under the impression of pulmonary edema with fluid overload, emergency hemodialysis was initiated after discussion with her family. Emergency intermittent hemodialysis was performed one time to remove $2,100 \mathrm{~mL}$ volume, but there was no substantial improvement on her oxygenation, which was $82 \%$ at the end of hemodialysis. There was no evidence of infection developed, including fever, cough with purulent sputum, pyuria, dysuria, or intra-abdominal pain. In addition, there was no new drug administered in the days preceding the second transfusion. Hemodynamic monitoring (PiCCO, Pulsion Medical Systems, Munich, Germany) after hemodialysis showed low cardiac index of 


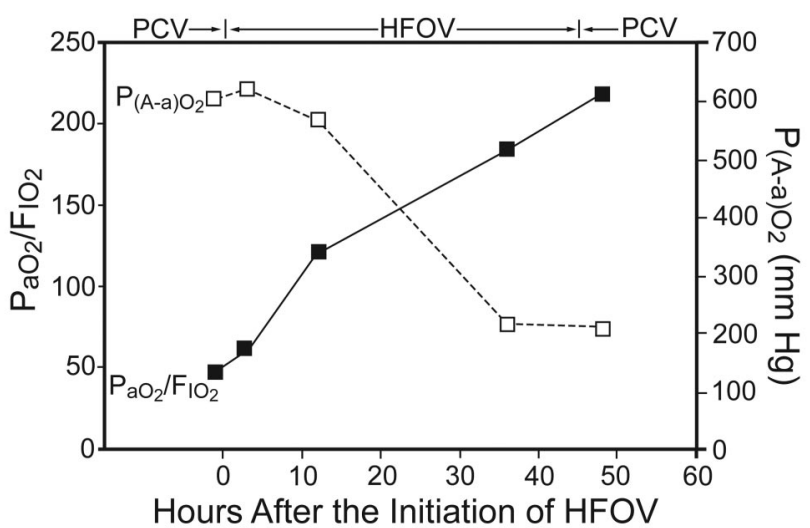

Fig. 2. The $\mathrm{P}_{\mathrm{aO}_{2}} / \mathrm{F}_{\mathrm{IO}_{2}}$ and alveolar-arterial oxygen difference $\left(\mathrm{P}_{(\mathrm{A}-\mathrm{a}) \mathrm{O}_{2}}\right)$ before and after high-frequency oscillatory ventilation (HFOV). $\mathrm{PCV}=$ pressure control ventilation.

$2.77 \mathrm{~L} / \mathrm{min} / \mathrm{m}^{2}$, low global end-diastolic volume index of $606 \mathrm{~mL} / \mathrm{m}^{2}$, low intrathoracic blood volume index of $757 \mathrm{~mL} / \mathrm{m}^{2}$, high extra-vascular lung water index of $37 \mathrm{~mL} /$ $\mathrm{kg}$, and high pulmonary vascular permeability index of 8.2. These data indicated non-cardiogenic pulmonary edema, and TRALI was diagnosed.

The initial mechanical ventilator setting consisted of a tidal volume of $6 \mathrm{~mL} / \mathrm{kg}$ of predicted body weight, ventilation rate 25 breaths/min, PEEP $12 \mathrm{~cm} \mathrm{H}_{2} \mathrm{O}$, and $100 \%$ oxygen. The PEEP level was gradually increased by $2 \mathrm{~cm} \mathrm{H}_{2} \mathrm{O}$, due to $\mathrm{S}_{\mathrm{pO}_{2}}$ below $90 \%$. After increasing PEEP level to $18 \mathrm{~cm} \mathrm{H}_{2} \mathrm{O}$, the maximum plateau pressure was $32 \mathrm{~cm} \mathrm{H}_{2} \mathrm{O}$. A recruitment maneuver was performed as a sustained inflation with mean airway pressure of $45 \mathrm{~cm} \mathrm{H}_{2} \mathrm{O}$ for 45 seconds before HFOV, but in vain. Due to persistent hypoxemia, despite 12 hours of pressure control ventilation support with $100 \%$ oxygen and $18 \mathrm{~cm} \mathrm{H}_{2} \mathrm{O}$ PEEP, the ventilator was changed to HFOV (3100B, SensorMedics, Yorba Linda, California). The initial ventilatory rate of $\mathrm{HFOV}$ was $3.5 \mathrm{~Hz}$, with mean airway pressure set at $30 \mathrm{~cm} \mathrm{H}_{2} \mathrm{O}$. Oxygenation improved with $\mathrm{HFOV}$, and the $\mathrm{F}_{\mathrm{IO}_{2}}$ decreased gradually to $45 \%$ with adequate arterial oxygen saturation. During HFOV the hemodialysis was not continued. The daily net intake/ output volume state was positive $300 \mathrm{~mL}$, and positive $250 \mathrm{~mL}$ during these $2 \mathrm{HFOV}$ days. The $\mathrm{P}_{\mathrm{aO}_{2}} / \mathrm{F}_{\mathrm{IO}_{2}}$ and alveolar-arterial oxygen difference before and after HFOV are shown in Figure 2. Mechanical ventilation was changed to conventional pressure control ventilation after 2 days of HFOV, and chest radiograph showed improvement of the diffuse bilateral pulmonary infiltration (see Fig. 1B).

The patient received 2 weeks of mechanical ventilation. Ten days after extubation she was transferred to the nephrology ward in stable respiratory condition.

\section{Discussion}

TRALI is a form of ALI defined as the presence of ALI within 6 hours of transfusion, in the absence of other risk factors for ALI. ${ }^{6}$ Critically ill patients with gastrointestinal bleeding are at a risk of developing TRALI, especially those with prior end-stage liver disease. ${ }^{7}$ The exact mechanism is unknown, although there is rising evidence that TRALI is immune-mediated. Its pathogenesis has been implied as 2 immunologic triggers: the infusion of antibodies directed against a cognate antigen in the recipient, and the infusion of biological response modifiers. ${ }^{8}$ In this case the first transfusion with packed RBC was tolerated without any complication. However, 6 days later, the ALI developed after the second transfusion with packed RBC. It may possibly be explained by the above 2-hit mechanism. In one of the largest series of TRALI cases reported, all of the patients required oxygen support, with $72 \%$ requiring mechanical ventilation. ${ }^{9}$ Nonetheless, $80 \%$ had substantial clinical improvement within 96 hours of onset of symptoms, while the remaining $20 \%$ either had a prolonged clinical course or succumbed to the disorder. The mortality rate is estimated to be between $5 \%$ and $15 \%$. Once clinical symptoms resolve, no permanent sequelae have been associated.

This young diabetic woman developed acute respiratory failure with severe hypoxemia $\left(\mathrm{P}_{\mathrm{aO}_{2}} / \mathrm{F}_{\mathrm{IO}_{2}} 132 \mathrm{~mm} \mathrm{Hg}\right)$ and diffuse bilateral infiltrates on chest radiograph $30 \mathrm{~min}$ after second blood transfusion. The other risk factors for ALI or ARDS were excluded by clinical history and course. In TRALI, patients are more likely to exhibit hypotension, low pulmonary arterial occlusion pressure, and unresponsiveness to diuretics, which indicate non-cardiogenic pulmonary edema. ${ }^{10,11}$ The TRALI diagnosis hinges on exclusion of other entities, including transfusion-associated circulatory overload. There is no sentinel characteristic that distinguishes TRALI from transfusion-associated circulatory overload. However, the clinical symptoms and signs, fluid status, cardiac function (including measurement of brain natriuretic peptide), and leukocyte antibody testing are applied to differentiate these 2 disorders. ${ }^{8}$ In this case, although we did not undertake serum brain natriuretic peptide level and serological examination such as human leukocyte antigen class I or II antibodies or neutrophil specific antibodies, hemodynamic monitoring showed low cardiac index, low global end-diastolic volume index, low intrathoracic blood volume index, high extra-vascular lung water index, and high pulmonary vascular permeability index. These data indicated noncardiogenic pulmonary edema, and TRALI was diagnosed.

For ALI and ARDS patients, lung-protective strategies with small tidal volume and adequate PEEP, as investigated in the ARDS Network trial, are associated with significantly more ventilator- and organ-failure-free days. 
There is also a $22 \%$ reduction in mortality, compared to higher tidal volumes. ${ }^{12}$ Management of TRALI is largely supportive, with more than $70 \%$ requiring mechanical ventilation. ${ }^{2,13}$ However, mechanical ventilation may be a risk factor for the onset of TRALI and may aggravate the injury by the use of injurious ventilator settings as shown in an animal study. ${ }^{14}$ For severe TRALI patients, strategies other than conventional mechanical ventilation, including prone position, nitric oxide inhalation, or extracorporeal membrane oxygenation, have been successfully applied to improve oxygenation. ${ }^{15-17}$ However, there is no report of HFOV in severe TRALI patients.

Theoretically, HFOV can achieve the goals of a lungprotective strategy. ${ }^{18}$ It can deliver very small tidal volumes at extremely rapid rates, thus avoiding large alveolar pressures and volume excursions typical of conventional ventilation. In addition, HFOV applied at a relatively high mean airway pressure can maintain lung requirements more effectively than PEEP levels that are typically set during conventional mechanical ventilation. Some studies of HFOV use for adult ARDS patients with severe hypoxemia and high plateau airway pressures have verified significant improvement in oxygenation and suggest that early initiation may have better outcomes. ${ }^{3,4,19}$ It has also been shown that HFOV is effective and safe in correcting oxygenation failure associated with ARDS in surgical patients. ${ }^{20}$

In this severe TRALI case, severe hypoxemia persisted despite pressure control ventilation support with $100 \%$ oxygen and PEEP of $18 \mathrm{~cm} \mathrm{H}_{2} \mathrm{O}$ for 12 hours. After shifting the conventional mechanical ventilation to HFOV with higher mean airway pressure, the refractory hypoxemia was effectively corrected and the $\mathrm{P}_{\mathrm{aO}_{2}} / \mathrm{F}_{\mathrm{IO}_{2}}$ gradually improved from 50 to $200 \mathrm{~mm} \mathrm{Hg}$. After 50 hours the patient was successfully weaned from HFOV to conventional mechanical ventilation. Currently, HFOV for adult ARDS is considered a rescue therapy if conventional mechanical ventilation fails. However, the timing of HFOV initiation remains controversial. Furthermore, the optimal lung-protective HFOV strategy has not been investigated, and there is no validated standard algorithm proposed for patients receiving HFOV.

The majority of TRALI cases are self-limited and will resolve spontaneously with adequate support. Here we report a case of severe TRALI successfully supported by HFOV after conventional mechanical ventilation failed. We suggest that HFOV may be considered earlier as a rescue therapy for severe TRALI patients not responding to conventional mechanical ventilation.

\section{REFERENCES}

1. Boshkov LK. Transfusion-related acute lung injury and the ICU. Crit Care Clin 2005;21(3):479-495.
2. Wallis JP. Transfusion-related acute lung injury (TRALI): presentation, epidemiology and treatment. Intensive Care Med 2007;33 (Suppl 1):S12-S16.

3. Mehta S, Lapinsky SE, Hallett DC, Merker D, Groll RJ, Cooper AB, et al. Prospective trial of high-frequency oscillation in adults with acute respiratory distress syndrome. Crit Care Med 2001;29(7):1360-1369.

4. Ferguson ND, Chiche JD, Kacmarek RM, Hallett DC, Mehta S, Findlay GP, et al. Combining high-frequency oscillatory ventilation and recruitment maneuvers in adults with early acute respiratory distress syndrome: the treatment with oscillation and an open lung strategy (TOOLS) trial pilot study. Crit Care Med 2005;33(3):479-486.

5. Sud S, Sud M, Friedrich JO, Meade MO, Ferguson ND, Wunsch H, et al. High frequency oscillation in patients with acute lung injury and acute respiratory distress syndrome (ARDS): systematic review and meta-analysis. BMJ 2010;340(5):c2327.

6. Toy P, Popovsky MA, Abraham E, Ambruso DR, Holness LG, Kopko PM, et al. Transfusion-related acute lung injury: definition and review. Crit Care Med 2005;33(4):721-726.

7. Benson AB, Austin GL, Berg M, McFann KK, Thomas S, Ramirez $\mathrm{G}$, et al. Transfusion-related acute lung injury in ICU patients admitted with gastrointestinal bleeding. Intensive Care Med 2010; 36(10):1710-1717.

8. Silliman CC. The two-event model of transfusion-related acute lung injury. Crit Care Med 2006;34(5 Suppl):S124-S131.

9. Popovsky MA, Moore SB. Diagnostic and pathogenetic considerations in transfusion-related acute lung injury. Transfusion 1985; 25(6):573-577.

10. Skeate RC, Eastlund T, Skeate RC, Eastlund T. Distinguishing between transfusion-related acute lung injury and transfusion associated circulatory overload. Curr Opin Hematol 2007;14(6):682-687.

11. Kleinman S, Caulfield T, Chan P, Davenport R, McFarland J, McPhedran S, et al. Toward an understanding of transfusion-related acute lung injury: statement of a consensus panel. Transfusion 2004; 44(12):1774-1789.

12. The Acute Respiratory Distress Syndrome Network. Ventilation with lower tidal volumes as compared with traditional tidal volumes for acute lung injury and the acute respiratory distress syndrome. N Engl J Med 2000;342(18):1301-1308.

13. Goldman M, Webert KE, Arnold DM, Freedman J, Blajchman MA. Proceedings of a consensus conference: towards an understanding of TRALI. Transfus Med Rev 2005;19(1):2-31.

14. Vlaar AP, Wolthuis EK, Hofstra JJ, Roelofs JJ, Boon L, Schultz MJ, Lutter R, Juffermans NP. Mechanical ventilation aggravates transfusion-related acute lung injury induced by MHC-I class antibodies. Intensive Care Med 2010;36(5):879-887.

15. Lin Y, Kanani N, Naughton F, Pendergrast J, Karkouti K. Case report: transfusion-related acute lung injury (TRALI)—a clear and present danger. Can J Anaesth 2007;54(12):1011-1016.

16. Cantlay K, Clarke M, Wallis J, Cosgrove JF. Immediate postoperative prone ventilation in the management of transfusion-related acute lung injury (TRALI) following infra-renal abdominal aortic aneurysm repair. Anaesth Intensive Care 2006;34(4):521-522.

17. Nouraei SM, Wallis JP, Bolton D, HasanA. Management of transfusion-related acute lung injury with extra-corporeal cardiopulmonary support in a four-year-old child. Br J Anaesth 2003;91(2): 292-294.

18. Fessler HE, Hess DR. Does high-frequency ventilation offer benefits over conventional ventilation in adult patients with acute respiratory distress syndrome? Respir Care 2007;52(5):595-608.

19. Chan KP, Stewart TE, Mehta S. High-frequency oscillatory ventilation for adult patients with ARDS. Chest 2007;131(6):1907-1916.

20. Kao KC, Tsai YH, Wu YK, Huang CT, Shih MJ, Huang CC. High frequency oscillatory ventilation for surgical patients with acute respiratory distress syndrome. J Trauma 2006;61(4):837-843. 